\title{
Aluminium-enriched metal-poor stars buried in the inner Galaxy
}

\author{
José G. Fernández-Trincado ${ }^{1,2}$, Timothy C. Beers ${ }^{3}$, Dante Minniti ${ }^{4,5}$, Baitian Tang ${ }^{6}$, Sandro Villanova ${ }^{7}$, \\ Doug Geisler ${ }^{7,8,9}$, Angeles Pérez-Villegas ${ }^{10}$, and Katherine Vieira ${ }^{1}$ \\ 1 Instituto de Astronomía y Ciencias Planetarias, Universidad de Atacama, Copayapu 485, Copiapó, Chile \\ e-mail: jose.fernandez@uda.cl \\ 2 Institut Utinam, CNRS-UMR 6213, Université Bourgogne-Franche-Comté, OSU THETA Franche-Comté, \\ Observatoire de Besançon, BP 1615, 251010 Besançon Cedex, France \\ ${ }^{3}$ Department of Physics and JINA Center for the Evolution of the Elements, University of Notre Dame, Notre Dame, IN 46556, \\ USA \\ ${ }^{4}$ Depto. de Cs. Físicas, Facultad de Ciencias Exactas, Universidad Andrés Bello, Av. Fernández Concha 700, Las Condes, Santiago, \\ Chile \\ 5 Vatican Observatory, 00120 Vatican City State, Italy \\ 6 School of Physics and Astronomy, Sun Yat-sen University, Zhuhai 519082, PR China \\ 7 Departamento de Astronomía, Universidad de Concepción, Casilla 160-C, Concepción, Chile \\ 8 Departamento de Astronomía, Universidad de La Serena, Avenida Juan Cisternas 1200, La Serena, Chile \\ 9 Instituto de Investigación Multidisciplinario en Ciencia y Tecnología, Universidad de La Serena, Benavente 980, La Serena, Chile \\ 10 Universidade de São Paulo, IAG, Rua do Matão 1226, Cidade Universitária, São Paulo 05508-900, Brazil
}

Received 18 August 2020 / Accepted 2 October 2020

\section{ABSTRACT}

\begin{abstract}
Stars with higher levels of aluminium and nitrogen enrichment are often key pieces in the chemical makeup of multiple populations in almost all globular clusters (GCs). There is also compelling observational evidence that some Galactic components could be partially built from dissipated GCs. The identification of such stars among metal-poor field stars may therefore provide insight into the composite nature of the Milky Way (MW) bulge and inner stellar halo, and could also reveal other chemical peculiarities. Here, based on APOGEE spectra, we report the discovery of 29 mildly metal-poor $([\mathrm{Fe} / \mathrm{H}] \lesssim-0.7)$ stars with stellar atmospheres strongly enriched in aluminium (Al-rich stars: $[\mathrm{Al} / \mathrm{Fe}] \gtrsim+0.5$ ), well above the typical Galactic levels, located within the solar radius toward the bulge region, which lies in highly eccentric orbits $(e \gtrsim 0.6)$. We find many similarities for almost all of the chemical species measured in this work with the chemical patterns of GCs, and therefore we propose that they have likely been dynamically ejected into the bulge and inner halo from GCs formed in situ and/or GCs formed in different progenitors of known merger events experienced by the MW, such as the Gaia-Sausage-Enceladus and/or Sequoia.
\end{abstract}

Key words. stars: abundances - stars: chemically peculiar - Galaxy: bulge - globular clusters: general - Galaxy: stellar content Galaxy: kinematics and dynamics

\section{Introduction}

The current standard picture of the inner region of our Galaxy suggests that it is made up of a complex variety of stellar populations, each with a characteristic structure, chemistry, and kinematics (see, e.g. Recio-Blanco et al. 2017; Queiroz et al. 2020a; Rojas-Arriagada et al. 2020, and references therein).

Every stellar population retains traces of the early phases of the formation and subsequent evolution of the inner disc, the massive bar structure $\left(\sim 10^{10} M_{\odot}\right)$ (Bland-Hawthorn \& Gerhard 2016), the pseudo-bulge, and a possible classical bulge (e.g. Combes et al. 1990; Minniti 1996; Athanassoula 2005; Zoccali et al. 2008; Barbuy et al. 2018), which is likely to be the product of mergers with and accretion of primordial globular clusters (GCs) or dwarf galaxies (e.g. Belokurov et al. 2018; Koppelman et al. 2019; Massari et al. 2019; Souza et al. 2020; Naidu et al. 2020). While our knowledge of the nature of the bulge has improved substantially in recent years, some key questions remain. In particular, some of the stars seen in the bulge today in the lower-metallicity range $(-2.0<[\mathrm{Fe} / \mathrm{H}]<-0.7)$ exhibit chemical compositions and kinematics that are different from those of the more metal-rich stars, and could contain information regarding the earliest phases of formation and evolution of the inner Galaxy (see, e.g. Fernández-Trincado et al. 2019a,b, 2020a).

A major advancement in revealing the nature of the low-metallicity stars $([\mathrm{Fe} / \mathrm{H}] \lesssim-0.7)$ in the bulge region was achieved by the discovery of a large population of giant stars with nitrogen over-abundances (N-rich; Schiavon et al. 2017; Fernández-Trincado et al. 2019b) and low- $\alpha$ stars (see, e.g. Recio-Blanco et al. 2017), which mimic the typical chemical patterns only seen in the so-called second-generation ${ }^{1} \mathrm{GC}$ stars. The origin of the N-rich stars (NRSs) remains controversial and is still a matter of debate (see Bekki 2019, for an alternative view). Recent studies propose the existence of stars that have lower aluminium enrichment $([\mathrm{Al} / \mathrm{Fe}]<+0.2)$ within $\sim 3.5 \mathrm{kpc}$ of

\footnotetext{
1 Second-generation (2G) is used here to refer to stars in GCs that display altered light-element abundances (e.g. He, C, N, O, Na, Al, and $\mathrm{Mg}$ ), which are different from those of typical MW field stars.
} 
the Galactic centre (Barbuy et al. 2018), with chemical patterns that resemble those of low-mass satellite galaxies of the Milky Way (MW; Das et al. 2020).

Taking advantage of high-resolution near-infrared (NIR) spectroscopy from the Apache Point Observatory Galactic Evolution Experiment (APOGEE-2; Majewski et al. 2017), we select some key elements ( $\mathrm{N}, \mathrm{Al}, \mathrm{Mg}, \mathrm{Si}$, among others) for investigation of any possible chemical peculiarities that could be used to constrain scenarios of their origin and relation to the massassembly history of the Galaxy (see, e.g. Fernández-Trincado et al. 2016, 2017, 2019a,b,c,d, 2020b).

In this Letter, we report the discovery of a unique collection of aluminium-enhanced (Al-rich) stars among a population of metal-poor ones. These stars exhibit $\mathrm{Al}$ abundances well above typical Galactic levels $([\mathrm{Al} / \mathrm{Fe}] \gtrsim+0.5)$ over a range of metallicities, and resemble those known for a long time to exist in Galactic GCs (see, e.g. Mészáros et al. 2020). However, they are unlikely to be linked to the accretion of dwarf galaxies, for which larger $\mathrm{Al}$ abundance ratios have not been observed to date (Hasselquist et al. 2019, and references therein). Observations and sample selection is described in Sect. 2. Results are discussed in Sect. 3. Finally, our conclusions are summarised in Sect. 4.

\section{Observations}

This work makes use of data primarily from the second generation of the Apache Point Observatory Galactic Evolution Experiment (APOGEE-2), which has now collected high-resolution $(R \sim 22500) H$-band spectra (NIR, $\sim 15145 \AA$ to $16960 \AA$, vacuum wavelengths) for almost 470000 sources in their sixteenth data release (APOGEE DR16: Ahumada et al. 2020), as part of the Sloan Digital Sky Survey IV (Blanton et al. 2017). For details of the sample selection, see Appendix A.

As we focus on selecting dissolved and/or evaporated GC stars in $[\mathrm{Al} / \mathrm{Fe}]$ space, we selected stars among metal-poor ones with aluminium enrichments greater than those expected for dwarf galaxies (see, e.g. Hasselquist et al. 2019) and MW stars, namely, $[\mathrm{Al} / \mathrm{Fe}] \gtrsim+0.5$. Our final sample comprises $29 \mathrm{Al}$-rich stars. With the uncalibrated ASPCAP stellar parameters fixed $\left(T_{\text {eff }}, \log g\right.$, and $\left.[\mathrm{M} / \mathrm{H}]\right)$, we derive, where possible, the abundances of $\mathrm{C}, \mathrm{N}, \mathrm{O}, \mathrm{Mg}, \mathrm{Al}, \mathrm{Si}$, and $\mathrm{Ce}$, as well as the metallicity for each Al-rich star. The derived abundances and kinematic parameters for this sample are presented in Tables B.1 and C.1.

\section{Elemental abundance analysis}

Large amounts of aluminium were found in the atmospheres of 29 previously unidentified metal-poor field giant stars. Our sample includes seven stars with orbital apocentres $\left(r_{\text {apo }}\right) \lesssim 3.5 \mathrm{kpc}$ (see Sect. 3.6), placing them well within the bulge region (see, e.g. Barbuy et al. 2018), and two halo interlopers located in the inner region of the Galaxy, which we refer to here as the bulge sample. The remaining stars in our sample are located within $\sim 13 \mathrm{kpc}$ from the Galactic centre. The light elements $(\mathrm{C}$, $\mathrm{N})$, the $\alpha$ elements $(\mathrm{O}, \mathrm{Mg}, \mathrm{Si})$, the odd-Z element $(\mathrm{Al})$, the iron-peak element ( $\mathrm{Fe})$, and the $s$-process element $(\mathrm{Ce})$ of these stars are compared to those for GC stars from Mészáros et al. (2020), and the local MW disc, halo, and bulge population from APOGEE DR16 (Ahumada et al. 2020). Abundances were determined from a $\chi^{2}$ minimisation of synthesised spectra using the BACCHUS code.

It is also important to note that there are no known GCs within an angular separation of $0.5^{\circ}$ for most of our stars, except for one (2M18035017-2552334), which is located $\sim 1.2 r_{\mathrm{t}}$ from the GC Terzan 10 . However, its $[\mathrm{Fe} / \mathrm{H}]$ and RV deviate significantly from the nominal parameters of the cluster, and therefore it cannot be considered a potential extra-tidal star candidate, as was recently found for other bulge GCs (NGC 6723, FernándezTrincado et al. 2020c).

Overall, we find that the elemental abundances of our sample are separated relatively cleanly from other stellar populations in different chemical dimensions, such as $[\mathrm{Mg}, \mathrm{N} / \mathrm{Fe}]-$ $[\mathrm{Al} / \mathrm{Fe}]$ planes. Results shown in Fig. 1 reveal that our observed stars differ substantially from those of the so-called N-rich (or NRS) bulge population (see, e.g. Schiavon et al. 2017) and from the rest of the entire $\mathrm{MW} /$ bulge sample, but exhibit a unique chemical signature similar to that of some GC $2 \mathrm{G}$ stars with higher $[\mathrm{Al} / \mathrm{Fe}]$. Also, their higher aluminium enrichment $([\mathrm{Al} / \mathrm{Fe}] \gtrsim+0.5)$ over a wide range of metallicities makes them unlikely to be associated to the typical $\mathrm{Al}$ enrichment seen in dwarf galaxies (Hasselquist et al. 2019), unless they are part of partially dissipated GCs in dwarf galaxies (see FernándezTrincado et al. 2020d).

\subsection{The iron-peak element: Fe}

Regarding the iron-peak element $(\mathrm{Fe})$, we find that our sample spans a wide range of iron abundances, $-1.68 \lesssim[\mathrm{Fe} / \mathrm{H}] \lesssim-0.76$ (see Fig. 1), suggesting that these stars were formed from different progenitors or in an unusual system like $\omega$ Cen (unless they are part of a binary system). It is interesting to note that our Al-rich stars toward the bulge region peak around $[\mathrm{Fe} / \mathrm{H}] \sim-1$, in the same way as NRS stars located in bulge (Schiavon et al. 2017), suggesting an association to them. It is therefore possible that we have identified a subfamily of that population which is more strongly enriched in aluminium, whilst the stars in our sample located outside the bulge region do not peak at any particular metallicity and are moderately more metal-poor.

\subsection{The $\alpha$-elements: $\mathrm{O}, \mathrm{Mg}$, and $\mathrm{Si}$}

Figure 1c shows that the $[\mathrm{O} / \mathrm{Fe}]$ abundance ratio of our sample is similar to that of the NRS, and MW/bulge stars at $[\mathrm{Fe} / \mathrm{H}] \gtrsim-1.5$, but is moderately enhanced toward the bulge region at metallicities as low as $[\mathrm{Fe} / \mathrm{H}] \lesssim-1.5$, and slightly enhanced for our sample outside the bulge region. In the same figures it is clear that the $[\mathrm{O} / \mathrm{Fe}]$ abundances of the Al-rich stars are on average consistent with the GC population at similar metallicity, and that these stars show a large dispersion in $[\mathrm{O} / \mathrm{Fe}]$ similar to that observed in GCs.

Regarding magnesium (see Fig. 1d), most of our targets exhibit low levels $([\mathrm{Mg} / \mathrm{Fe}] \lesssim+0.2)$ and behave like accreted halo stars in the low-Mg sequence. At higher metallicity $([\mathrm{Fe} / \mathrm{H}] \gtrsim-1.25)$, a few stars show enhancement in $[\mathrm{Mg} / \mathrm{Fe}]$ relative to the disc, providing evidence that these stars do not belong to the canonical disc or halo of the MW, or to the bulge population, and have lower $[\mathrm{Mg} / \mathrm{Fe}]$ than the NRS on average. On the other hand the $[\mathrm{Mg} / \mathrm{Fe}]$ levels and the star-to-star dispersion observed in our sample is consistent with that observed in GC stars and $\omega$ Cen stars.

The $\alpha$-element $\mathrm{Si}$ is found to be higher in our sample compared to the NRS and MW/bulge stars, but similar to those known to exist in $\omega$ Cen stars, and a few other Galactic GCs at similar metallicity. The slightly higher $[\mathrm{Si} / \mathrm{Fe}](\sim+0.25)$ of our stars indicate that the initial composition of the gas that formed them was slightly different from the NRS and MW stars, but similar to that of GC stars, providing further evidence that the Al-rich stars could be likely accreted stars from a progenitor whose chemical history is similar to that of known Galactic GCs. 
a)
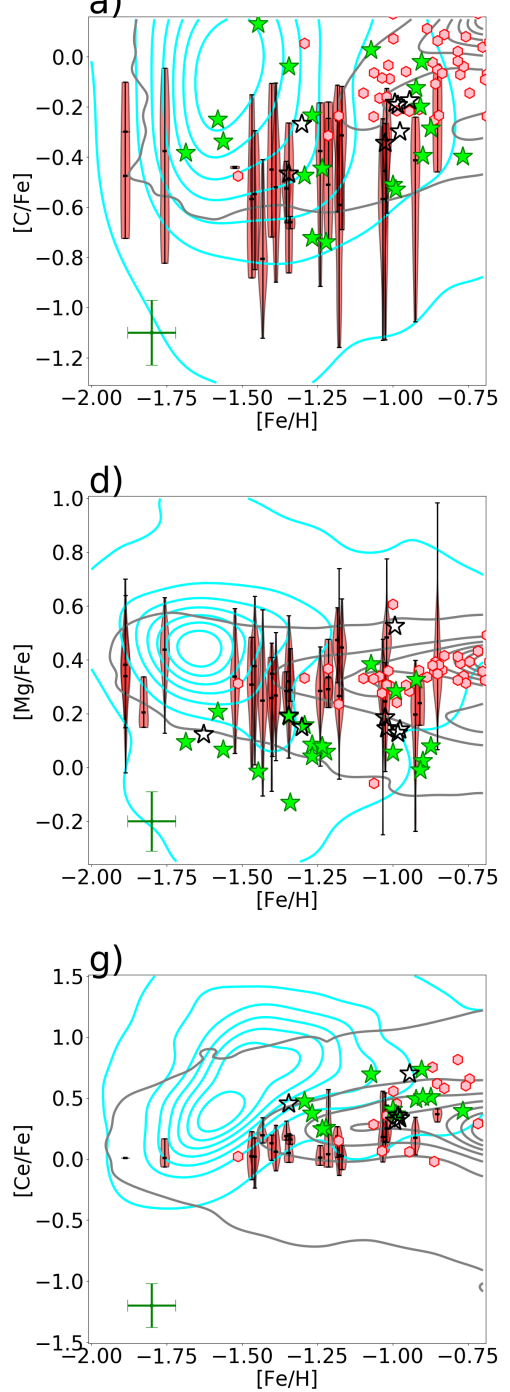

b)

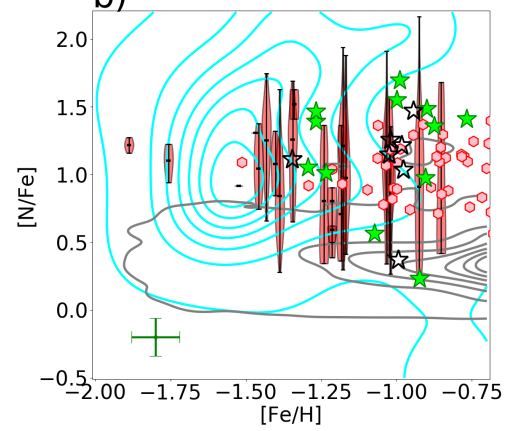

e)

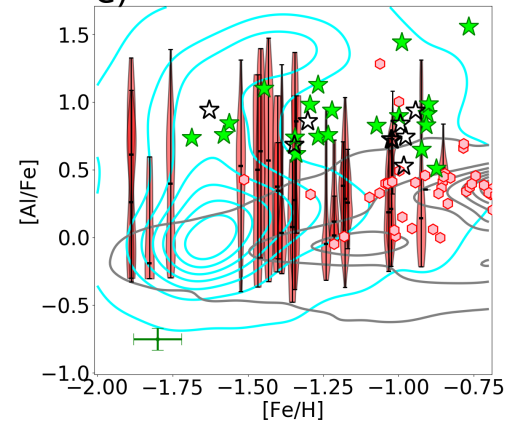

h)

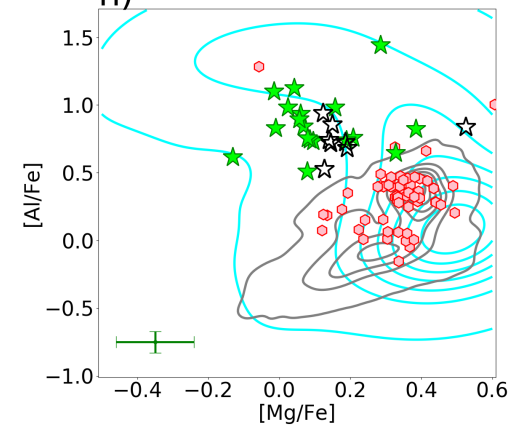

C)
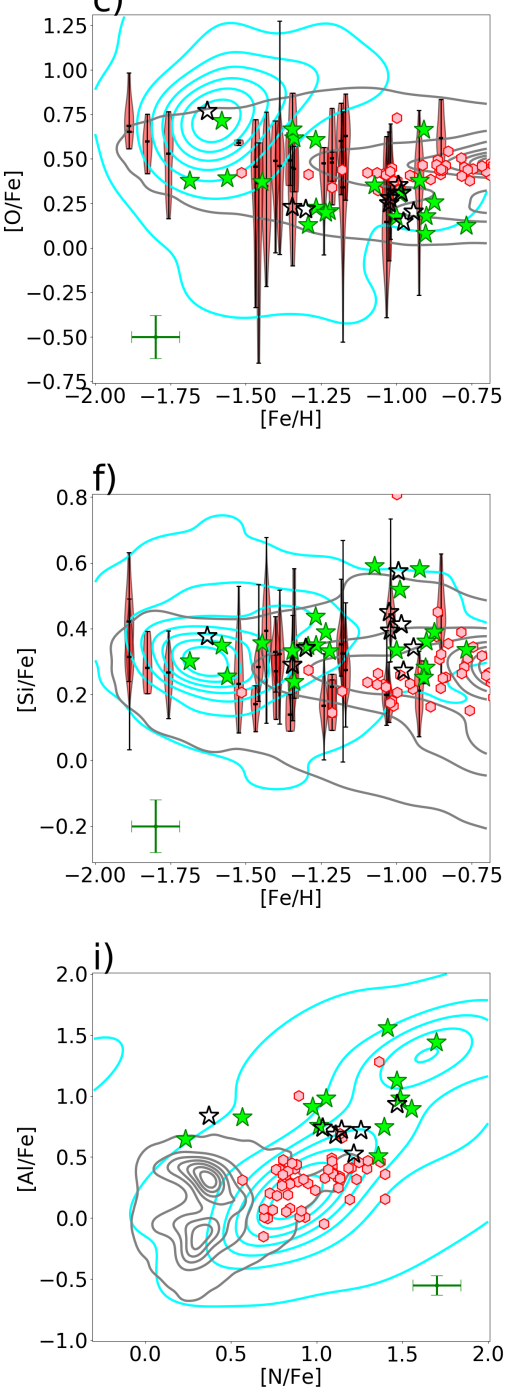

Fig. 1. Kernel density estimation of $[\mathrm{X} / \mathrm{Fe}]$ (with $\mathrm{X}=\mathrm{C}, \mathrm{N}, \mathrm{O}, \mathrm{Mg}, \mathrm{Al}, \mathrm{Si}$, or $\mathrm{Ce}$ ) with metallicity $(a-g)$, and $[\mathrm{Al} / \mathrm{Fe}]$ as a function of $[\mathrm{Mg}, \mathrm{N} / \mathrm{Fe}]$ ratios $(i, h)$ for the APOGEE DR16 stars (grey contours) surviving the quality cuts discussed in Sect. 2 and corresponding to the chemical domain of the halo, and low-metallicity end of the bulge and disc, corrected by the offsets between pipelines (see Fig. D.2). $\omega$ Centauri stars (cyan contours) from Mészáros et al. (2020) are also plotted for comparison. The black and lime star symbols refer to Al-rich stars in the bulge sample and outside the bulge region, respectively. Our sample is compared to GCs (crimson violin representation, indicating with horizontal lines the median and limits of the distribution; left to right: M 53, NGC 6397, NGC 5466, M 55, M 22, M 79, NGC 6752, M 13, M 2, NGC 6544, M 3, M 54, M 10, NGC 6522, NGC 3201, NGC 6229, Pal 5, NGC 288, M 5, M 12, NGC 1851, NGC 362, M 4, NGC 2808, Pal 6, and M 107) from Mészáros et al. (2020), and a sample of selected NRS (pink hexagons) from Schiavon et al. (2017) with available APOGEE DR16 abundance ratios. The plotted error bars (green symbols) show the typical uncertainties associated with our sample.

\subsection{The light elements: $C$ and $N$}

The outlying stars with high levels of $\mathrm{Al}$ enhancement are also enhanced in $\mathrm{N}$, well above the Galactic levels $([\mathrm{N} / \mathrm{Fe}] \gtrsim+0.8)$ as can be seen in Figs. 1b and i, except for three stars at metallicities above $[\mathrm{Fe} / \mathrm{H}] \gtrsim-1.2$ that exhibit nitrogen abundance lower than $[\mathrm{N} / \mathrm{Fe}] \sim+0.5$, but clearly distinguishable from the canonical Galactic components in the $[\mathrm{N} / \mathrm{Fe}]-[\mathrm{Al} / \mathrm{Fe}]$ plane. Furthermore, the Al-rich stars are slightly more enriched in nitrogen than the NRS, indicating that they do not necessarily share the same star-formation history, instead showing similarities to GC environments. Interestingly, we can see a clear $\mathrm{N}-\mathrm{Al}$ correlation in Fig. 1 between our Al-rich stars toward the bulge region and the NRS, which is strikingly similar to that seen in Galactic GCs like $\omega$ Cen.
Regarding carbon, we find similar patterns to NRS and GC populations at the same metallicities. The Al-rich stars from our sample have slightly lower levels than the bulge, canonical disc, and halo populations, extending down well below $[\mathrm{C} / \mathrm{Fe}] \lesssim-0.7 \mathrm{dex}$.

\subsection{The odd-Z elements: Al}

Figure $1 \mathrm{~h}$ shows a well-distinguished clump of Al-rich stars, differing from the NRS, MW, and bulge stars, indicating that these stars are likely members of a distinct population confined to the inner part of the Galaxy (based on their orbital parameters; see Sect. 3.6), possibly part of the innermost distribution of the stellar halo. There are also a few cases in the $[\mathrm{Mg} / \mathrm{Fe}]-[\mathrm{Al} / \mathrm{Fe}]$ plane with large $\mathrm{Al}$ enhancement paired with 
low $\mathrm{Mg},[\mathrm{Mg} / \mathrm{Fe}]<0$, which is a signature of GC $2 \mathrm{G}$ stars (see, e.g. Fernández-Trincado et al. 2017; Lucey et al. 2019).

Overall, Fig. 1h displays the chemical-abundance pattern of GC stars from $\omega$ Cen (Mészáros et al. 2020); this GC spans as wide a metallicity range as our observed Al-rich stars. The star-to-star scatter of the Al-rich stars for almost all the chemical species is similar to that typically found in $\omega$ Cen stars. The similarity between Al-rich stars and GC 2G stars can be further tested through examination of the $[\mathrm{Mg}, \mathrm{Al} / \mathrm{Fe}]-[\mathrm{Al} / \mathrm{Fe}]$ planes. Interestingly, we find that Al-rich stars exhibit similar characteristics to the NRSs, that is, they also show high [N/Fe] correlated with $[\mathrm{Al} / \mathrm{Fe}]$ and anti-correlated with $[\mathrm{C} / \mathrm{Fe}]$. To first order, these branches strongly resemble those known for a long time to exist in GCs, and are not present in the Galactic field. As far as we know, this is the first time the existence of clear $\mathrm{Mg}-\mathrm{Al}$ anticorrelation and $\mathrm{N}-\mathrm{Al}$ correlation among metal-poor stars with chemical anomalies toward the Galactic bulge is reported, and beyond the GC environments where these have typically been identified so far. This finding reinforces the unique nature of our sample, and confirms the complex and composite structure of the MW bulge.

\subsection{The s-process element: $\mathrm{Ce}$}

The APOGEE DR16 [Ce/Fe] abundance ratio patterns (Cunha et al. 2017) are shown for MW stars in Fig. 1g and stars in the bulge region in Fig. 1g. Figure $1 \mathrm{~g}$ indicates that $[\mathrm{Ce} / \mathrm{Fe}]$ is slightly more enhanced than in NRSs, GCs, or MW stars, but agrees with GC stars (Mészáros et al. 2020) at similar metallicities. However, there is currently no explanation for this unexpected tendency.

\subsection{Orbital analysis}

The model and distribution of orbital parameters are presented in Appendix C. Overall, we find that all the stars in our sample have highly eccentric orbits $(e \gtrsim 0.6)$. Figure C. 1 shows the Galactic spatial distribution and the orbital elements for the Al-rich stars. As is clear from the figure, the majority of the stars are found to have radial ( $r_{\text {per }} \lesssim 3.5 \mathrm{kpc}$ ) orbits in different configurations (e.g. retrograde, prograde, and $\mathrm{P}-\mathrm{R}^{2}$ orbits). The orbital elements also reveal that most of the Al-rich stars are currently located near their apocentric distances $\left(r_{\text {apo }}\right)$, placing many of them within the solar radius ( $r_{\text {gal }} \lesssim 8 \mathrm{kpc}$ ) and inside of the bulge region $\left(r_{\text {gal }} \lesssim 3 \mathrm{kpc}\right.$ ), with rather small excursions above the Galactic plane $\left(|Z|_{\max } \lesssim 3 \mathrm{kpc}\right)$. Several (11 out of 29 stars) of them have larger $\left(3 \mathrm{kpc} \lesssim|Z|_{\max } \lesssim 25 \mathrm{kpc}\right.$ ) vertical excursions, making them halo interlopers within the solar radius. We conclude that these stars live in the bulge region and the inner stellar halo of the Galaxy, respectively.

An interesting aspect of the Al-rich stars can be seen in Fig. C.1h. Strikingly, this figure reveals that a disc control sample taken from APOGEE DR16 populates the top part of the $v_{\phi}$ versus $v_{\mathrm{R}}$ distribution, whilst the Al-rich stars fall in the bottom part, where the Gaia-Sausage-Enceladus (GSE), Helmi Streams (Helmi St.), and the Arjuna+Sequoia+I'itoi (ASI) halo structures reside (see, e.g. Myeong et al. 2018; Naidu et al. 2020), which are dominated by metal-poor $(-3 \lesssim[\mathrm{Fe} / \mathrm{H}] \lesssim-0.5)$ stars. For comparison, we calculated the orbital solutions for Galactic GCs from Baumgardt et al. (2019) and adopted the progenitor classification of Massari et al. (2019). Figure C.2

\footnotetext{
2 We refer to prograde-retrograde (P-R) orbits as the ones that flip their sense from prograde to retrograde, or vice versa, along their orbit.
}

shows the characteristic orbital energy $\left(\left(E_{\max }+E_{\min }\right) / 2\right)$ versus the orbital Jacobi constant $\left(E_{\mathrm{J}}\right)$ distribution of Galactic GCs and our Al-rich stars. This diagram reveals that the Al-rich stars populate a wide range of energies, similar to that of Galactic GCs with different origins, suggesting that these Al-rich stars may have emerged from different GCs.

Are the Al-rich stars largely the signatures of tidal disruption of accreted dwarf galaxies? This perhaps seems unlikely, given that the observed Al-rich stars exhibit higher $[\mathrm{Al} / \mathrm{Fe}] \gtrsim+0.5$, not observed in present-day dwarf galaxy stars (see Hasselquist et al. 2019, and references therein). Even though most of the Al-rich stars share similar orbital eccentricity, spatial distribution, and location in the $v_{\phi}$ versus $v_{\mathrm{R}}$ plane as those seen in high- $\alpha$ disc stars, in-situ halo stars, metal-poor thick-disc stars, Sagittarius-, Thamnos-, GSE-, Helmi St.-, and ASI-structures (Naidu et al. 2020), their association seems unlikely, given the higher $\mathrm{Al}$ and $\mathrm{N}$ abundances observed. Our sample is also not part of the unclassified debris stars reported in Naidu et al. (2020), as the Al-rich stars possess larger orbital eccentricities than these latter. However, as there are some GCs that appear to be possibly associated with some of the known mergers, in particular to GSE, the Helmi St., and Sequoia (see, e.g. Massari et al. 2019), it is possible that some of our observed stars could be associated with those merger events through their partially dissipated GCs as highlighted in Fig. C.2.

All the chemical and dynamical properties described above suggest that the Al-rich stars could be the signatures of the tidal disruption of GCs likely accreted and/or formed in situ. This population could have been dynamically ejected in different orbital configurations into the bulge region and the inner stellar halo from GC systems at similar metallicity, or possibly a massive system like $\omega$ Cen (Meza et al. 2005; Majewski et al. 2012).

\section{Conclusions}

We report the detection of aluminium-enriched $([\mathrm{Al} / \mathrm{Fe}] \gtrsim+0.5)$ giant stars toward the bulge and inner stellar halo of the Galaxy. The majority of the 29 stars of our sample have unique abundance patterns seen in accreted GCs, and dynamical properties consistent with the bulge and halo population.

The identification of such unusual stars toward the bulge region confirms the proposed composite nature of the MW bulge (e.g. Recio-Blanco et al. 2017; Rojas-Arriagada et al. 2020; Queiroz et al. 2020a). Our finding also reveals, for the first time, that there are many chemically anomalous metal-poor stars in the $v_{\phi}$ versus $v_{\mathrm{R}}$ plane residing in the same position where massive merger events (e.g. Gaia-Sausage-Enceladus and Sequoia) have been identified. However, our study also reveals that some $\alpha$ elements ( $\mathrm{Si}$ ) and odd-Z elements (Al) appear to rule out any direct link with those known merger events, but some indirect association through GCs may still apply.

We propose that many of our Al-rich stars were possible former members of several stellar clusters with similar chemical enrichment to that of GCs likely accreted and/or formed in situ. The peculiar chemical signature of these objects makes them excellent candidates to trace the fossil relic population of the early MW, which became part of the general stellar population.

Acknowledgements. The author is grateful for the enlightening feedback from the anonymous referee. J.G.F.-T. is supported by FONDECYT No. 3180210. T.C.B. acknowledges partial support for this work from grant PHY 14-30152: Physics Frontier Center/JINA Center for the Evolution of the Elements (JINACEE), awarded by the US National Science Foundation. D.M. is supported by the BASAL Center for Astrophysics and Associated Technologies (CATA) through 
grant AFB 170002, and by project FONDECYT Regular No. 1170121. B.T. gratefully acknowledges support from the National Natural Science Foundation of China under grant No. U1931102 and support from the hundred-talent project of Sun Yat-sen University. S.V. gratefully acknowledeges the support provided by Fondecyt regular n. 1170518. D.G. gratefully acknowledges support from the Chilean Centro de Excelencia en Astrofísica y Tecnologías Afines (CATA) BASAL grant AFB-170002. D.G. also acknowledges financial support from the Dirección de Investigación y Desarrollo de la Universidad de La Serena through the Programa de Incentivo a la Investigación de Académicos (PIA-DIDULS). A.P.-V. acknowledges FAPESP for the postdoctoral fellowship No. 2017/158931 and the DGAPA-PAPIIT grant IG100319. The SDSS-IV/APOGEE-2 survey made this study possible.

\section{References}

Ahumada, R., Prieto, C. A., Almeida, A., et al. 2020, ApJS, 249, 3

Athanassoula, E. 2005, MNRAS, 358, 1477

Barbuy, B., Chiappini, C., \& Gerhard, O. 2018, ARA\&A, 56, 223

Baumgardt, H., Hilker, M., Sollima, A., \& Bellini, A. 2019, MNRAS, 482, 5138

Bekki, K. 2019, MNRAS, 490, 4007

Belokurov, V., Erkal, D., Evans, N. W., Koposov, S. E., \& Deason, A. J. 2018, MNRAS, 478, 611

Bland-Hawthorn, J., \& Gerhard, O. 2016, ARA\&A, 54, 529

Blanton, M. R., Bershady, M. A., Abolfathi, B., et al. 2017, AJ, 154, 28

Bovy, J., Leung, H. W., Hunt, J. A. S., et al. 2019, MNRAS, 490, 4740

Bowen, I. S., \& Vaughan, A. H., 1973, Appl. Opt., 12, 1430

Carollo, D., Beers, T. C., Chiba, M., et al. 2010, ApJ, 712, 692

Combes, F., Debbasch, F., Friedli, D., \& Pfenniger, D. 1990, A\&A, 233, 82

Cunha, K., Smith, V. V., Hasselquist, S., et al. 2017, ApJ, 844, 145

Das, P., Hawkins, K., \& Jofré, P. 2020, MNRAS, 493, 5195

Fernández-Trincado, J. G., Robin, A. C., Moreno, E., et al. 2016, ApJ, 833, 132

Fernández-Trincado, J. G., Zamora, O., García-Hernández, D. A., et al. 2017, ApJ, 846, L2

Fernández-Trincado, J. G., Beers, T. C., Placco, V. M., et al. 2019a, ApJ, 886, L8

Fernández-Trincado, J. G., Beers, T. C., Tang, B., et al. 2019b, MNRAS, 488, 2864

Fernández-Trincado, J. G., Mennickent, R., Cabezas, M., et al. 2019c, A\&A, 631, A97

Fernández-Trincado, J. G., Zamora, O., Souto, D., et al. 2019d, A\&A, 627, A178

Fernández-Trincado, J. G., Chaves-Velasquez, L., Pérez-Villegas, A., et al. 2020a, MNRAS, 495, 4113

Fernández-Trincado, J. G., Minniti, D., Beers, T. C., et al. 2020b, A\&A, in press, https://doi .org/10.1051/0004-6361/202039328
Fernández-Trincado, J. G., Carigi, L., Beers, T. C., et al. 2020c, ApJ, submitted Fernández-Trincado, J. G., Beers, T. C., Minniti, D., et al. 2020d, ApJ, submitted [arXiv:2010.00024]

Gaia Collaboration (Brown, A. G. A., et al.) 2018, A\&A, 616, A1

García Pérez, A. E., Allende Prieto, C., Holtzman, J. A., et al. 2016, AJ, 151, 144

Gunn, J. E., Siegmund, W. A., Mannery, E. J., et al. 2006, AJ, 131, 2332

Gustafsson, B., Edvardsson, B., Eriksson, K., et al. 2008, A\&A, 486, 951

Hasselquist, S., Carlin, J. L., Holtzman, J. A., et al. 2019, ApJ, 872, 58

Holtzman, J. A., Hasselquist, S., Shetrone, M., et al. 2018, AJ, 156, 125

Jönsson, H., Allende Prieto, C., Holtzman, J. A., et al. 2018, AJ, 156, 126

Koppelman, H. H., Helmi, A., Massari, D., Roelenga, S., \& Bastian, U. 2019, A\&A, 625, A5

Lindegren, L., Hernández, J., Bombrun, A., et al. 2018, A\&A, 616, A2

Lucey, M., Hawkins, K., Ness, M., et al. 2019, MNRAS, 488, 2283

Majewski, S. R., Nidever, D. L., Smith, V. V., et al. 2012, ApJ, 747, L37

Majewski, S. R., Schiavon, R. P., Frinchaboy, P. M., et al. 2017, AJ, 154, 94

Martell, S. L., Shetrone, M. D., Lucatello, S., et al. 2016, ApJ, 825, 146

Massari, D., Koppelman, H. H., \& Helmi, A. 2019, A\&A, 630, L4

Masseron, T., Merle, T., \& Hawkins, K. 2016, Astrophysics Source Code Library [record ascl:1605.004]

Masseron, T., García-Hernández, D. A., Mészáros, S., et al. 2019, A\&A, 622, A191

Mészáros, S., Masseron, T., García-Hernández, D. A., et al. 2020, MNRAS, 492, 1641

Meza, A., Navarro, J. F., Abadi, M. G., \& Steinmetz, M. 2005, MNRAS, 359, 93

Minniti, D. 1996, ApJ, 459, 175

Myeong, G. C., Evans, N. W., Belokurov, V., Sanders, J. L., \& Koposov, S. E. 2018, ApJ, 863, L28

Naidu, R. P., Conroy, C., Bonaca, A., et al. 2020, ApJ, 901, 48

Nataf, D. M., Wyse, R. F. G., Schiavon, R. P., et al. 2019, AJ, 158, 14

Nidever, D. L., Holtzman, J. A., Allende Prieto, C., et al. 2015, AJ, 150, 173

Queiroz, A. B. A., Anders, F., Chiappini, C., et al. 2020a, A\&A, 638, A76

Queiroz, A. B. A., Chiappini, C., Perez-Villegas, A., et al. 2020b, A\&A, submitted [arXiv:2007.12915]

Recio-Blanco, A., Rojas-Arriagada, A., de Laverny, P., et al. 2017, A\&A, 602, L14

Rojas-Arriagada, A., Zasowski, G., Schultheis, M., et al. 2020, MNRAS, 499, 1037

Schiavon, R. P., Zamora, O., Carrera, R., et al. 2017, MNRAS, 465, 501

Souza, S. O., Kerber, L. O., Barbuy, B., et al. 2020, ApJ, 890, 38

Wilson, J. C., Hearty, F. R., Skrutskie, M. F., et al. 2019, PASP, 131, 055001

Zoccali, M., Hill, V., Lecureur, A., et al. 2008, A\&A, 486, 177 


\section{Appendix A: Sample selection}

APOGEE DR16 includes data taken from both the northern and southern hemisphere using the APOGEE spectrographs (Wilson et al. 2019) mounted on the $2.5 \mathrm{~m}$ Sloan Foundation telescope (Gunn et al. 2006) at Apache Point Observatory in New Mexico (APOGEE-2N: North, APO), and on the $2.5 \mathrm{~m}$ Irénée du Pont telescope (Bowen \& Vaughan 1973) at Las Campanas Observatory (APOGEE-2S: South, LCO) in Chile. For details regarding the APOGEE atmospheric parameter analysis we refer readers to the APOGEE Stellar Parameter and Chemical Abundances pipeline (ASPCAP: García Pérez et al. 2016), while for details of the grid of synthetic spectra and errors see Holtzman et al. (2018). We refer the reader to Nidever et al. (2015) for further details of the data-reduction pipeline for APOGEE. The model grids for APOGEE DR16 are based on a complete set of MARCS (Gustafsson et al. 2008) stellar atmospheres, which are now extended to effective temperatures $\left(T_{\text {eff }}\right)$ as low as $3200 \mathrm{~K}$.

The parent sample upon which this work is based is defined as follows. Metal-poor stars were selected from the DR16 cata$\operatorname{logue}^{3}$ that match the following criteria: (i) $-2 \lesssim[\mathrm{Fe} / \mathrm{H}] \lesssim-0.7$; imposing a lower limit on metallicity, i.e. $[\mathrm{Fe} / \mathrm{H}]>-2$, allows for the inclusion of metal-poor stars with high-quality spectra with reliable parameters and chemical abundances, while requiring stars with metallicity below $[\mathrm{Fe} / \mathrm{H}]=-0.7$ minimises the presence of disc stars; (ii) stars with $S / N>60$ were selected to ensure that we are selecting spectra that have wellknown uncertainties in their stellar parameters and chemical abundances, and to remove stars with lower quality spectra; (iii) $3200 \mathrm{~K}<T_{\text {eff }}<5500 \mathrm{~K}$, this temperature range ensures that stellar parameters are reliably and consistently determined, and maximises the overall quality of the abundances considered; (iv) the estimated surface gravity $(\log g$ ) must be less than 3.6, to ensure inclusion of giant and subgiant stars, which have more accurate ASPCAP parameters than the dwarf stars with $\log g>3.6$ (due to the lack of asteroseismic surface gravities for dwarfs, only stars with $\log g<3.6$ have calibrated surface gravities); (v) ASPCAPFLAG $==\mathbb{0}$; this cut ensures that there are no major flagged issues, i.e. low signal-to-noise ratio, poor synthetic spectral fit, stellar parameters near grid boundaries, among others; and (vi) stars belonging to Galactic GCs from Mészáros et al. (2020), anomalous stars from Martell et al. (2016), Schiavon et al. (2017), and Fernández-Trincado et al. (2016, 2017, 2019a,b,c,d), and stars with carbon abundances $[\mathrm{C} / \mathrm{Fe}] \gtrsim+0.15$ (to ensure the low carbon abundances typical of GC stars) were excluded from the sample.

The final selected sample amounts to a total of 13338 stars with high-quality parameters. We search for aluminium-enriched stars by carrying out a semi-independent stellar-abundance analysis of Al I lines using the BACCHUS ${ }^{4}$ code (Masseron et al. 2016), following the method described by Fernández-Trincado et al. (2019b).

\section{Appendix B: Basic parameters}

In Table B. 1 we provide the abundances of $\mathrm{C}, \mathrm{N}, \mathrm{O}, \mathrm{Mg}, \mathrm{Al}, \mathrm{Si}$, $\mathrm{Fe}$, and $\mathrm{Ce}$ for the 29 stars analysed in this work. The abundances were determined by making a careful line selection, and a lineby-line analysis in local thermodynamic equilibrium (LTE) with the BACCHUS code (Masseron et al. 2016). The $T_{\text {eff }}, \log g$, and overall metallicity $([\mathrm{M} / \mathrm{H}])$ have been fixed, and were selected from uncalibrated ASPCAP $T_{\text {eff }}, \log g$, and $[\mathrm{M} / \mathrm{H}]$ from DR16. In addition, lines were rejected if they were found to not be well-reproduced by the synthesis, or if they were flagged as problematic by the BACCHUS pipeline. None of the 29 Al-rich star candidates exhibit particularly strong variability in its radial velocity $\left(V_{\text {scatter }} \lesssim 0.5 \mathrm{~km} \mathrm{~s}^{-1}\right.$ ) over the period ( $\lesssim 6$ months) of the APOGEE observations, therefore with the existing data the observed abundance anomalies could not be explained by channels invoking binary mass transfer or stellar variability. The uncertainties in the elemental abundances were computed by adding the typical sensitivities of the abundance and the internal error in quadrature, in the same manner as described in Fernández-Trincado et al. (2019a,b,c,d). The typical uncertainties are shown with two crossed green error bars (one per axis plotted) in Fig. 1. 
Table B.1. Basic parameters of the Al-rich stars.

\begin{tabular}{|c|c|c|c|c|c|c|c|c|c|c|c|c|c|c|c|}
\hline APOGEE_ID & $\begin{array}{l}T_{\text {eff }} \\
(\mathrm{K})\end{array}$ & $\log g$ & {$[\mathrm{M} / \mathrm{H}]$} & $\begin{array}{c}\mu_{\mathrm{t}} \\
\left(\mathrm{km} \mathrm{s}^{-1}\right)\end{array}$ & $\begin{array}{c}V_{\text {scatter }} \\
\left(\mathrm{km} \mathrm{s}^{-1}\right)\end{array}$ & $\begin{array}{c}S / N \\
\left(\text { pixel }^{-1}\right)\end{array}$ & \#Visits & {$[\mathrm{C} / \mathrm{Fe}]$} & {$[\mathrm{N} / \mathrm{Fe}]$} & {$[\mathrm{O} / \mathrm{Fe}]$} & {$[\mathrm{Mg} / \mathrm{Fe}]$} & {$[\mathrm{Al} / \mathrm{Fe}]$} & {$[\mathrm{Si} / \mathrm{Fe}]$} & {$[\mathrm{Fe} / \mathrm{H}]$} & {$[\mathrm{Ce} / \mathrm{Fe}]$} \\
\hline $2 \mathrm{M} 16035902+0703353$ & 5095 & 2.71 & -1.34 & 0.6 & 0.13 & 147 & 6 & -0.03 & . & 0.66 & 0.18 & 0.73 & 0.33 & -1.34 & \\
\hline 2M17343682-2654206 & 4511 & 1.98 & -0.98 & 1.8 & 0.11 & 80 & 2 & $\ldots$ & 1.25 & 0.27 & 0.14 & 0.71 & 0.39 & -1.02 & 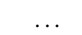 \\
\hline $2 \mathrm{M} 17254298-2106351$ & 4740 & 1.89 & -1.52 & 1.1 & 0.09 & 74 & 2 & & $\ldots$ & 0.76 & 0.12 & 0.94 & 0.37 & -1.62 & \\
\hline $2 \mathrm{M} 21372238+1244305$ & 5062 & 2.86 & -0.93 & 0.9 & 0.40 & 127 & 4 & -0.19 & & 0.66 & -0.01 & 0.82 & 0.25 & -0.90 & \\
\hline $2 \mathrm{M} 17461523-3407127$ & 4559 & 1.93 & -1.22 & 1.6 & $\ldots$ & 84 & 1 & -0.26 & $\ldots$ & 0.21 & 0.14 & 0.85 & 0.34 & -1.30 & 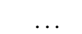 \\
\hline 2M17313399-2640506 & 3792 & 0.62 & -0.93 & 2.7 & 0.40 & 232 & 2 & -0.19 & 1.21 & 0.30 & 0.12 & 0.52 & 0.41 & -0.98 & 0.35 \\
\hline $2 \mathrm{M} 19010991-2121082$ & 4368 & 1.39 & -1.33 & 1.7 & 0.06 & 346 & 3 & -0.46 & 1.11 & 0.22 & 0.19 & 0.68 & 0.29 & -1.34 & 0.45 \\
\hline $2 \mathrm{M} 16443329+3624504$ & 4816 & 2.06 & -1.36 & 1.3 & 0.04 & 164 & 3 & 0.13 & $\ldots$ & 0.37 & -0.01 & 1.10 & 0.35 & -1.44 & $\ldots$ \\
\hline $2 \mathrm{M} 15535622+2237419$ & 4284 & 1.27 & -1.26 & 1.8 & 0.15 & 177 & 2 & -0.47 & 1.05 & 0.12 & 0.15 & 0.98 & 0.34 & -1.29 & 0.47 \\
\hline $2 \mathrm{M} 15053060+4211466$ & 4689 & 1.72 & -1.52 & 1.4 & 0.09 & 122 & 4 & -0.33 & & 0.39 & 0.06 & 0.84 & 0.25 & -1.56 & \\
\hline $2 \mathrm{M} 16282719-0050428$ & 4433 & 1.95 & -1.05 & 1.9 & 0.21 & 128 & 6 & 0.02 & 0.56 & 0.35 & 0.38 & 0.82 & 0.59 & -1.07 & 0.69 \\
\hline $2 \mathrm{M} 18041915-3703227$ & 3808 & 0.33 & -1.07 & 1.8 & 0.02 & 239 & 2 & -0.18 & 0.37 & 0.35 & 0.52 & 0.83 & 0.57 & -0.99 & 0.31 \\
\hline $2 \mathrm{M} 15532396-3509322$ & 4766 & 2.12 & -1.53 & 0.8 & 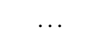 & 89 & 1 & -0.25 & & 0.71 & 0.20 & 0.75 & 0.34 & -1.58 & \\
\hline 2M17425767-2803437 & 4569 & 1.96 & -0.85 & 2.9 & 0.39 & 92 & 2 & -0.17 & 1.46 & 0.20 & & 0.93 & 0.34 & -0.94 & 0.69 \\
\hline 2M18490114-3042332 & 4426 & 1.79 & -0.96 & 2.1 & & 159 & 1 & -0.29 & 1.03 & 0.14 & 0.13 & 0.74 & 0.27 & -0.97 & 0.33 \\
\hline $2 \mathrm{M} 16055435+3003432$ & 4469 & 1.34 & -1.65 & 1.8 & 0.08 & 185 & 2 & -0.38 & $\ldots$ & 0.37 & 0.09 & 0.73 & 0.30 & -1.68 & . \\
\hline $2 \mathrm{M} 17254242-1331201$ & 4485 & 1.99 & -0.97 & 2.1 & 0.08 & 256 & 3 & -0.51 & 1.55 & 0.17 & 0.05 & 0.89 & 0.33 & -0.99 & 0.40 \\
\hline $2 \mathrm{M} 18524618-2520372$ & 4518 & 2.07 & -1.02 & 1.5 & & 70 & 1 & -0.34 & 1.14 & 0.24 & 0.18 & 0.72 & 0.44 & -1.02 & \\
\hline $2 \mathrm{M} 14063545+2734116$ & 4859 & 2.17 & -1.32 & 1.8 & 0.28 & 74 & 6 & & $\ldots$ & 0.61 & -0.13 & 0.61 & 0.23 & -1.34 & $\cdots$ \\
\hline $2 \mathrm{M} 16102218-2158014$ & 4342 & 1.55 & -1.24 & 2.1 & $\ldots$ & 326 & 1 & -0.72 & 1.39 & 0.22 & 0.08 & 0.74 & 0.35 & -1.26 & 0.37 \\
\hline $2 \mathrm{M} 02593571-1731458$ & 4472 & 2.09 & -0.96 & 1.3 & 0.46 & 87 & 3 & -0.12 & 0.23 & 0.37 & 0.32 & 0.64 & 0.58 & -0.92 & 0.49 \\
\hline $2 \mathrm{M} 22015198-1141479$ & 4269 & 1.57 & -1.25 & 1.8 & 0.15 & 279 & 3 & -0.44 & 1.01 & 0.19 & 0.07 & 0.75 & 0.39 & -1.23 & 0.24 \\
\hline $2 \mathrm{M} 16112935-2000538$ & 4871 & 2.35 & -1.20 & 1.1 & $\ldots$ & 117 & 1 & -0.23 & 1.46 & 0.60 & 0.04 & 1.12 & 0.43 & -1.26 & \\
\hline $2 \mathrm{M} 06572697+5543115$ & 4776 & 2.22 & -1.21 & 1.2 & 0.02 & 446 & 3 & -0.73 & & 0.20 & 0.05 & 0.93 & 0.33 & -1.22 & 0.25 \\
\hline $2 \mathrm{M} 17431262-2843363$ & 4149 & 0.16 & -0.72 & 2.0 & $\ldots$ & 115 & 1 & -0.39 & 1.41 & 0.12 & $\ldots$ & 1.55 & 0.33 & -0.76 & 0.39 \\
\hline $2 \mathrm{M} 17475763-2913591$ & 3837 & 0.06 & -1.04 & 2.0 & $\ldots$ & 100 & 1 & -0.52 & 1.69 & 0.30 & 0.28 & 1.44 & 0.51 & -0.98 & 0.36 \\
\hline $2 \mathrm{M} 17480799-2712083$ & 3913 & 1.11 & -0.74 & 2.9 & $\ldots$ & 90 & 1 & -0.28 & 1.35 & 0.25 & 0.07 & 0.50 & 0.38 & -0.87 & 0.50 \\
\hline 2M17484544-2915516 & 4241 & 1.53 & -0.82 & 2.9 & 0.02 & 81 & 2 & -0.01 & 0.97 & 0.08 & $\ldots$ & 0.91 & 0.28 & -0.90 & 0.73 \\
\hline 2M18035017-2552334 & 4190 & 1.59 & -0.88 & 2.3 & $\ldots$ & 137 & 1 & -0.39 & 1.48 & 0.17 & 0.02 & 0.98 & 0.36 & -0.90 & 0.50 \\
\hline
\end{tabular}

\section{Appendix C: Galactic orbits}

The basic kinematic parameters for the 29 stars and their respective orbital elements are listed in Table C.1. Since the true Galactic potential is not accurately known in the inner Galaxy, and the results of our simulations may depend significantly on the assumed parameters, we also run the simulations with a slowand fast-rotating bar, i.e. $31 \mathrm{~km} \mathrm{~s}^{-1}$ and $51 \mathrm{~km} \mathrm{~s}^{-1}$, respectively, and see how it affects the derived orbital parameters of the stars. The value inside parentheses in Table C.1 show the effects in the orbital elements by adopting different angular velocity for the Galactic bar. The individual variations are within typical errors of the ensemble of orbits with $\Omega_{\mathrm{bar}}=41 \mathrm{~km} \mathrm{~s}^{-1}$, which do not change the overall results from our sample.

We used the GravPot $16^{5}$ model to study the orbital history of our sample. The orbital integration scheme follows the methodology introduced in Fernández-Trincado et al. (2020a), except for the angular velocity of the bar, for which we adopted $\Omega_{\mathrm{bar}}=41 \mathrm{~km} \mathrm{~s}^{-1} \mathrm{kpc}^{-1}$ (see, e.g. Bovy et al. 2019). The model has been rescaled to the Sun's Galactocentric dis- tance, $8 \mathrm{kpc}$, and the velocity of the Local Standard of Rest, $v_{\text {LSR }}=244.5 \mathrm{~km} \mathrm{~s}^{-1}$.

For each star, we integrated an ensemble of orbits over a 2 Gyr time-span. The uncertainties in the input data were randomly propagated as $1 \sigma$ variations in a Gaussian Monte Carlo re-sampling (e.g. Fernández-Trincado et al. 2020a). The resulting values were estimated as the median of 1000 realisations, and the error distributions were estimated by finding the 16th and 84th percentiles. In addition, we also obtained the change in the orbital elements when the bar angular velocity is varied by $\pm 10 \mathrm{~km} \mathrm{~s}^{-1} \mathrm{kpc}$ (values inside parentheses in Table C.1). Figure C.1 follows the orbital elements of our sample.

We use the APOGEE DR16 distances deduced from the StarHorse code (Queiroz et al. 2020b,a), radial velocities from the APOGEE survey, and absolute proper motions from the Gaia DR2 catalogue (Gaia Collaboration 2018). Only sources with "good" astrometry were considered in our orbit computations, i.e. stars with renormalised unit weight error, RUWE $<1.4$ (see, e.g. Lindegren et al. 2018). A total of 24 out of 29 stars in our sample meet this criterion.

\footnotetext{
5 https://gravpot.utinam.cnrs.fr
} 
a)

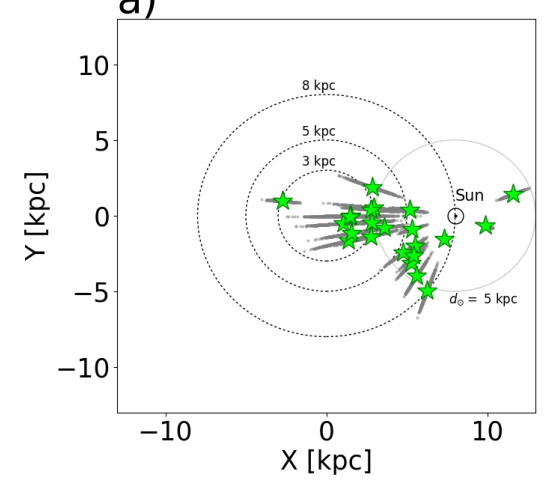

d)
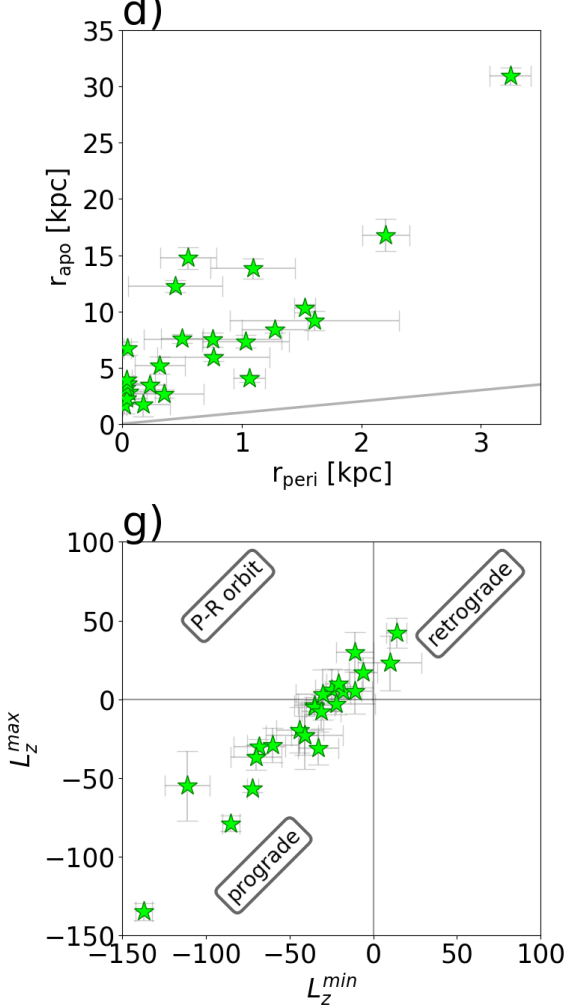

b)

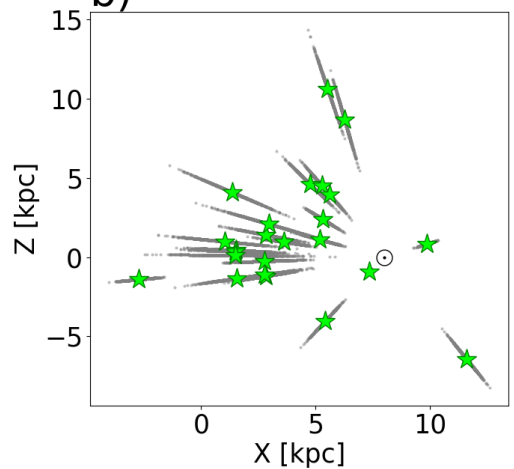

e)

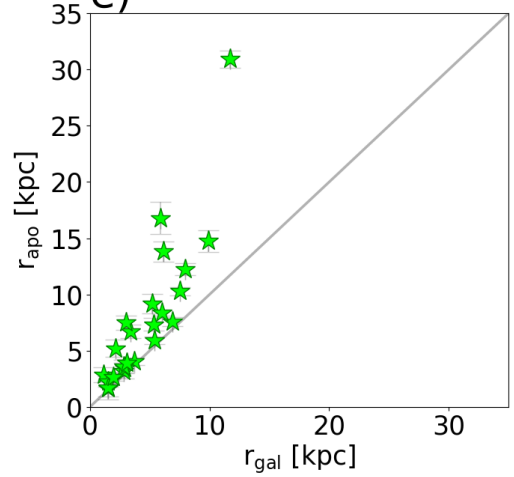

h)

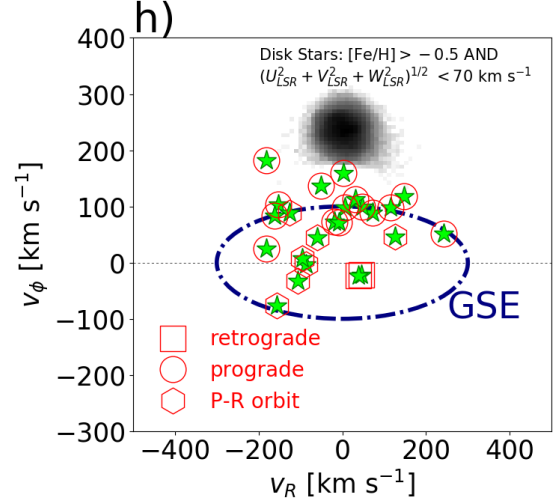

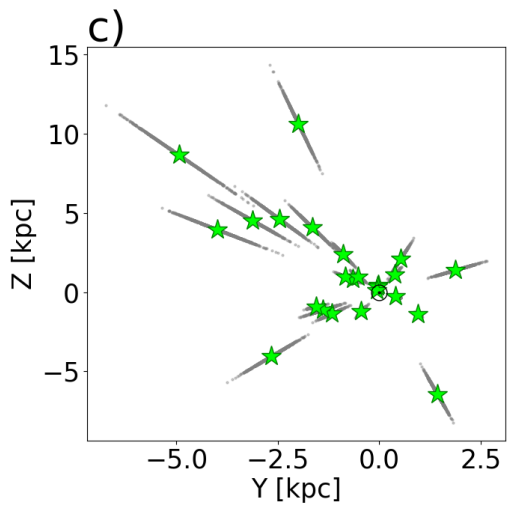

f)

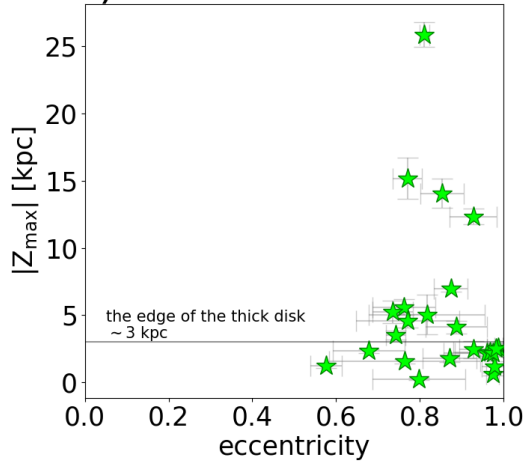

Fig. C.1. Panels $a-c$ : Galactic location of the Al-rich stars (lime star symbols). The aligned grey points show the results of 1000 Monte Carlo resampling incorporating measurement errors. The orbital elements are shown in panels $d-g$. The straight lines in panels $d$ and $e$ indicate the one-to-one line. For panel $d$, a star on this line would have a circular orbit, and in panel $e$, a star on this line is being observed at apocentre. The horizontal line in panel $f$ indicates the approximate edge of the thick disc according to Carollo et al. (2010). The type of orbital configuration of each star is shown in panel $g$. The median of the orbital parameters for each star is shown with their respective 16th and 84th percentiles (error bars) obtained for the model with the bar having $\Omega_{\mathrm{bar}}=41 \mathrm{~km} \mathrm{~s}^{-1} \mathrm{kpc}^{-1}$. The distribution of the velocity components $v_{\theta}$ vs. $v_{\mathrm{R}}$ for the studied Al-rich stars is shown in panel $h$. The blue dashed line represents the approximate region for stars associated with Gaia-Sausage in $v_{\theta}$ vs. $v_{\mathrm{R}}$ space based on Belokurov et al. (2018). In addition, a 2D heat map (grey) of the densely distributed disc stars from APOGEE DR16 is shown for comparison. The unfilled red symbols in panel $h$ mark the type of orbital configuration. 
J. G. Fernández-Trincado et al.: Fossil relics of early Milky Way

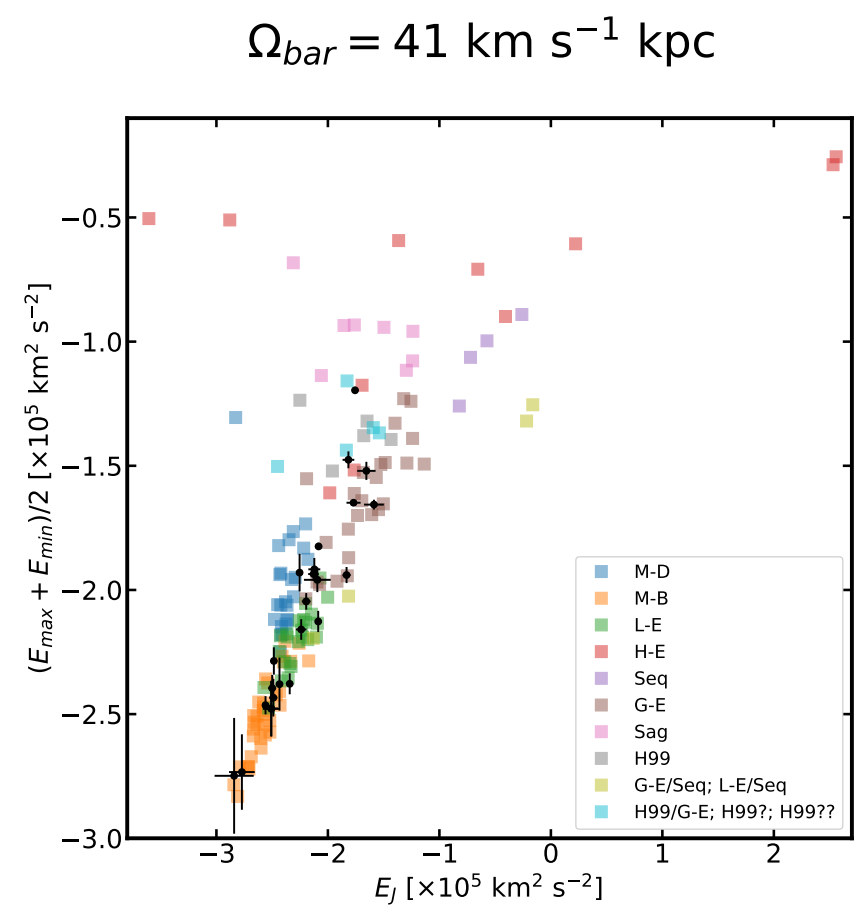

Fig. C.2. Characteristic orbital energy $\left(\left(E_{\max }+E_{\min }\right) / 2\right)$ versus the orbital Jacobi constant $\left(E_{\mathrm{J}}\right)$ in the non-inertial reference frame where the bar is at rest. Square symbols refer to Galactic GCs, colour-coded according to their association with different progenitors from Massari et al. (2019). The black dots with error bars refer to the Al-rich star analysed in this study. 


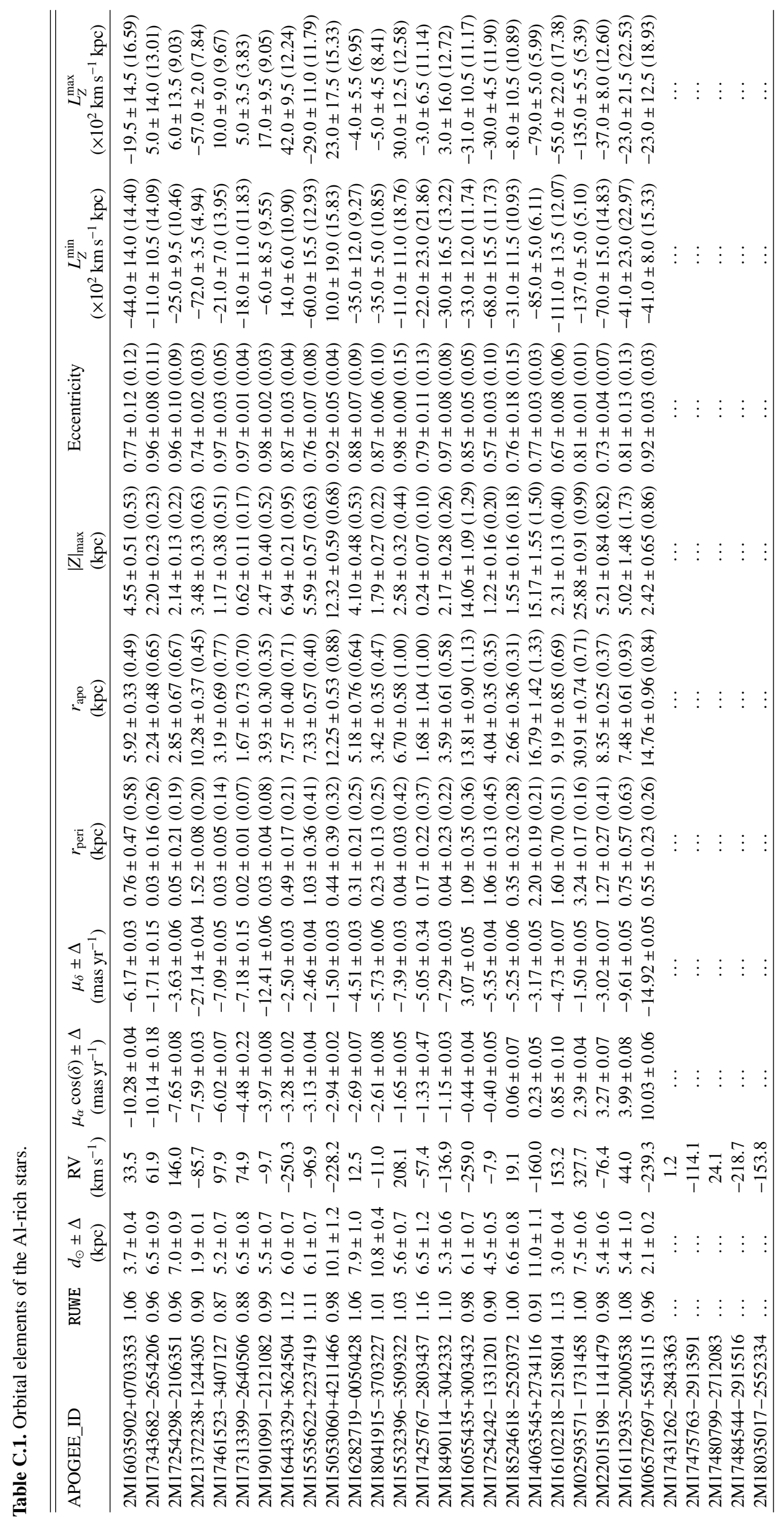




\section{Appendix D: Comparisons to ASPCAP}

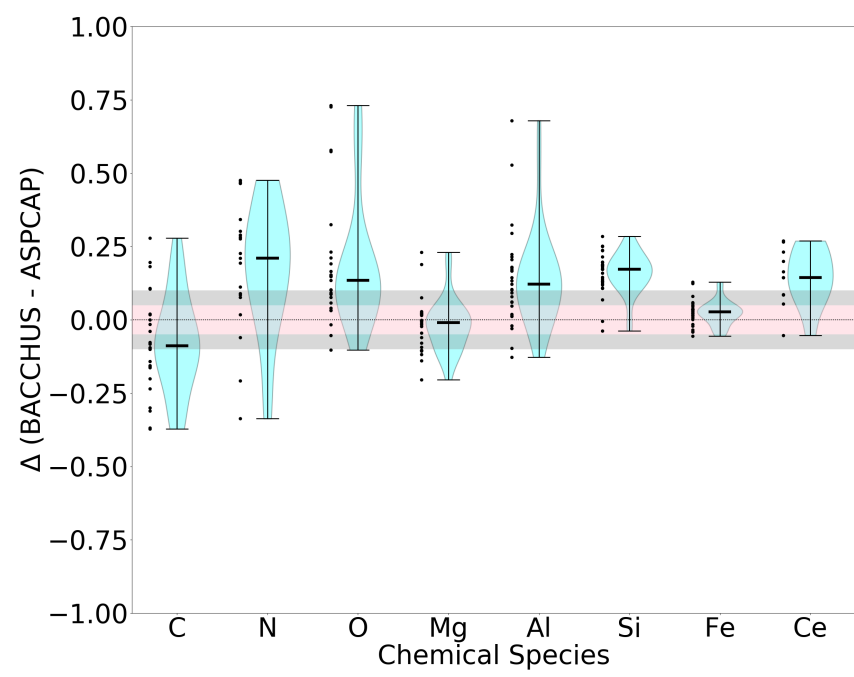

Fig. D.1. Violin diagram showing the differences between the derived elemental abundances between our analysis with the BACCHUS code against those values of the ASPCAP pipeline for our 29 Al-rich stars. Each violin representation indicates with horizontal lines the median and limits of the distribution. The horizontal shaded regions show the typical errors of $+0.05 \mathrm{dex}$, and $+0.1 \mathrm{dex}$ found by the ASPCAP pipeline.

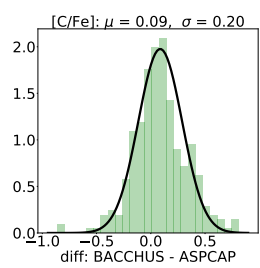

${ }_{5}[\mathrm{Mg} / \mathrm{Fe}]: \mu=0.07, \sigma=0.08$
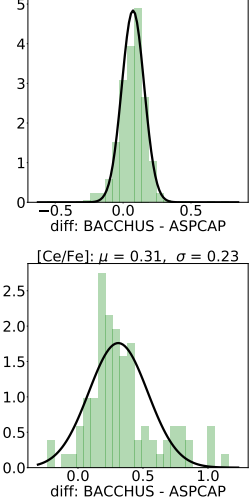
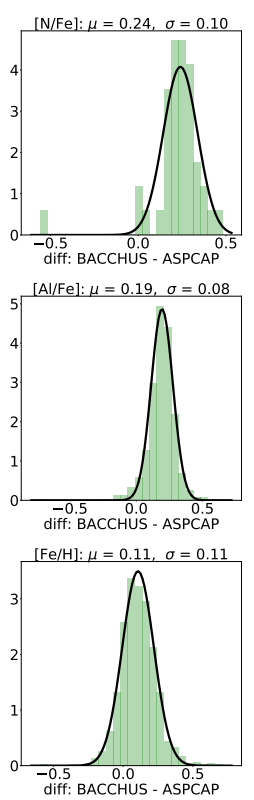

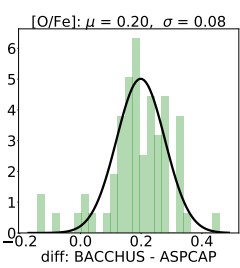

[Si/Fe]: $\mu=0.05, \sigma=0.09$

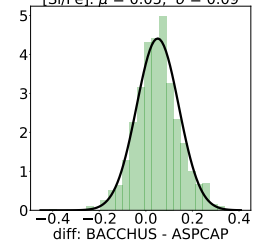

Fig. D.2. Differences in abundances produced by the BACCHUS and ASPCAP for a control sample of $\sim 1000$ giants belonging to the main body of the MW (halo, disc and bulge), at the same metallicity range as examined in this study. The numbers in each title of each panel indicate the peak $(\mu)$ and the standard deviation $(\sigma)$ of the fitted normal distribution, showing that ASPCAP values are significantly offset from the BACCHUS ones.
Figure D.1 compares our abundance determinations with those of APOGEE DR16 (Ahumada et al. 2020). The differences are shown as a density estimation in a violin representation, whereby the median, minimum, and maximum differences of each chemical species are marked. We find that the $[\mathrm{Fe} / \mathrm{H}]$ metallicities we derive are in excellent agreement with ASPCAP pipeline, with a median distribution slightly higher than the reported by DR16, but within the typical error $( \pm 0.05 \mathrm{dex})$ determined by the ASPCAP pipeline. Furthermore, this indicates that we reproduce the precision of the ASPCAP pipeline for the iron lines. However, there are significant differences in the range of certain abundance ratios comparing BACCHUS to ASPCAP. For example, $[\mathrm{N}, \mathrm{O}, \mathrm{Al}$, $\mathrm{Si}, \mathrm{Ce} / \mathrm{Fe}]$ ratios derived with BACCHUS are $\sim 0.1-0.75$ dex higher than the ASPCAP values, while $[\mathrm{O}, \mathrm{Mg} / \mathrm{Fe}]$ are $\sim 0.05-0.4 \mathrm{dex}$ lower than ASPCAP determinations, possibly due to some issues with the accuracy (zero-point) of ASPCAP abundances, limits of the model grid (Jönsson et al. 2018), and/or the difficulty of fitting lines where the intensity is comparable to the variance. For a more detailed discussion, we refer the reader to Masseron et al. (2019). While the ASPCAP pipeline uses a global fit to the continuum in the three detector chips independently, the BACCHUS pipeline places the pseudo-continuum in a region around the lines of interest. We believe that our manual method is more reliable, since it avoids possible shifts in the continuum location due to imperfections in the spectral subtraction along the full spectral range.

Figure D. 2 shows the typical offset of each chemical species between the BACCHUS and ASPCAP pipeline for a control sample of $\sim 1000$ metal-poor stars $(-2 \lesssim[\mathrm{Fe} / \mathrm{H}] \sim-0.7)$ belonging to the main components of the MW (halo, disc and bulge). We find that ASPCAP significantly underestimates most of the chemical species by about $\sim 0.1$ to 0.3 dex for most of the metal-poor stars (although see Nataf et al. 2019, for an alternative view). Such offsets were taken into consideration for the whole MW stars presented in Fig. 1.

\section{Appendix E: Al I features}

Figure E.1 provides a brief examination of typical $H$-band spectra for five Al-rich stars and Al-normal stars that exhibit clear Al I spectral absorption features. This figure reassures us of the existence of a real chemical peculiarity in the rich stars. The spectra of these stars are compared in a wavelength range containing several $\mathrm{Al}$ I lines, i.e. at 1.67189, 1.67505 and $1.67633 \mu \mathrm{m}$, which are indicated by the cyan shadow region. Remarkably, the Al-rich stars have relatively strong Al I lines compared to Al-normal stars with the same atmospheric parameters. This can only be attributed to a difference in aluminium abundance. 

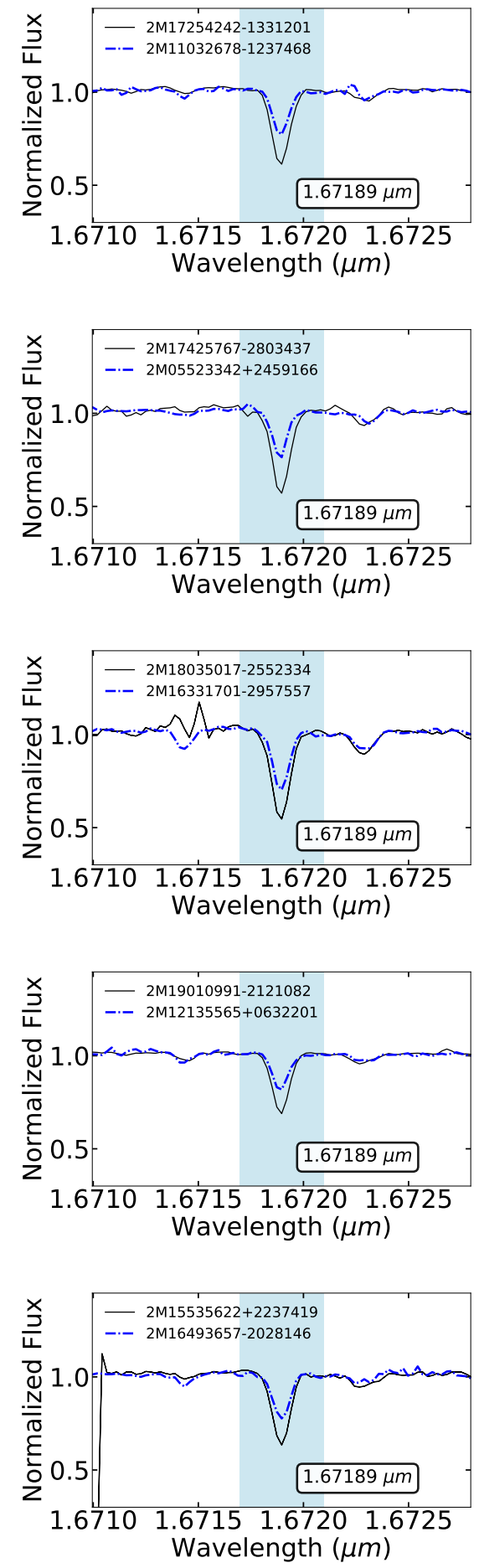
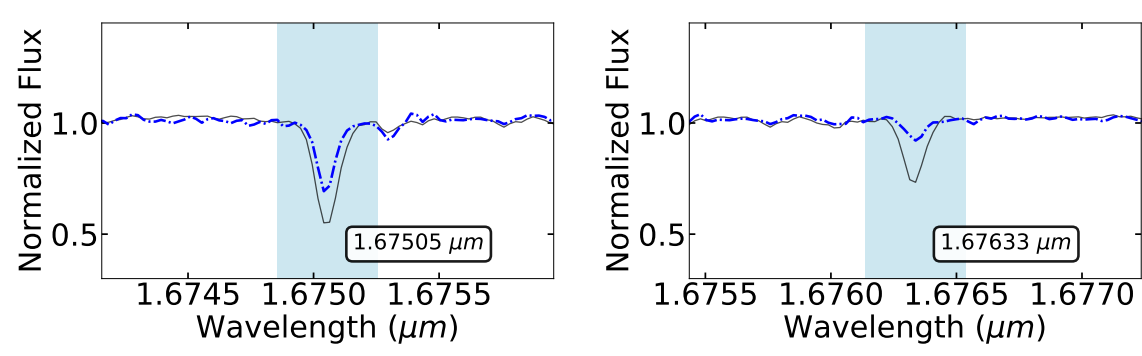

$\begin{array}{llll}1.6755 & 1.6760 & 1.6765 & 1.6770\end{array}$ Wavelength $(\mu \mathrm{m})$
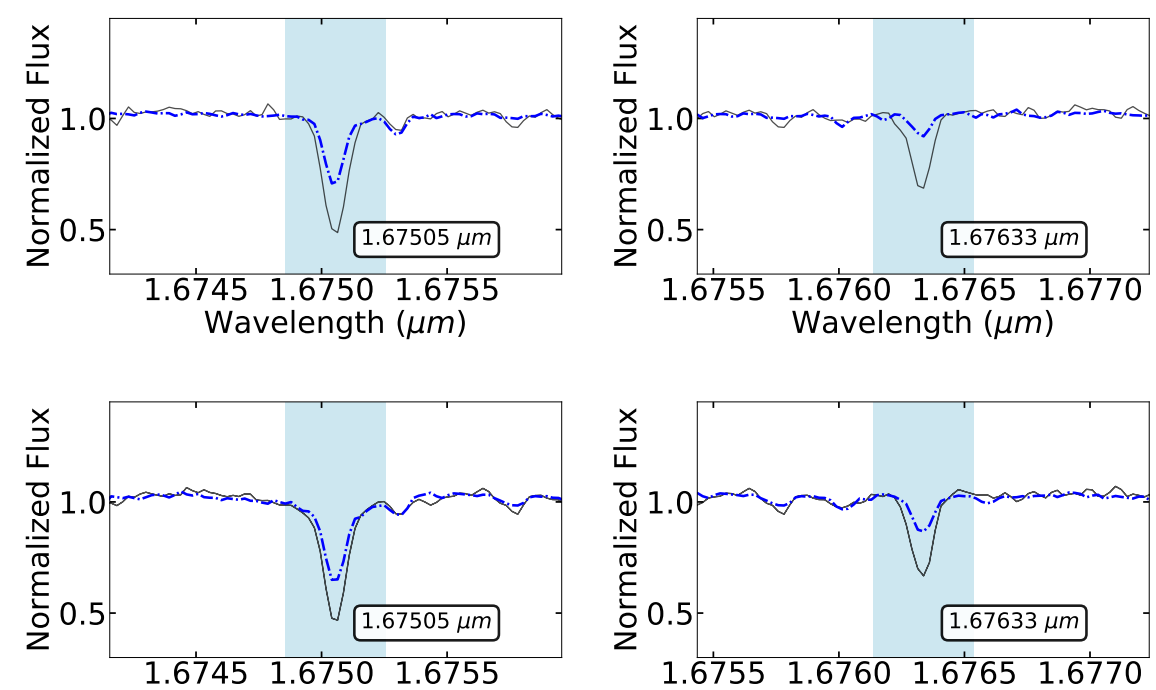

Wavelength $(\mu \mathrm{m})$

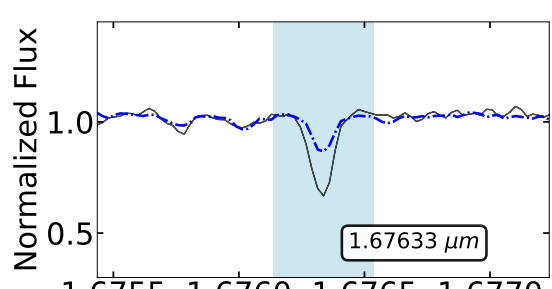

$\begin{array}{llll}1.6755 & 1.6760 & 1.6765 \quad 1.6770\end{array}$ Wavelength $(\mu \mathrm{m})$
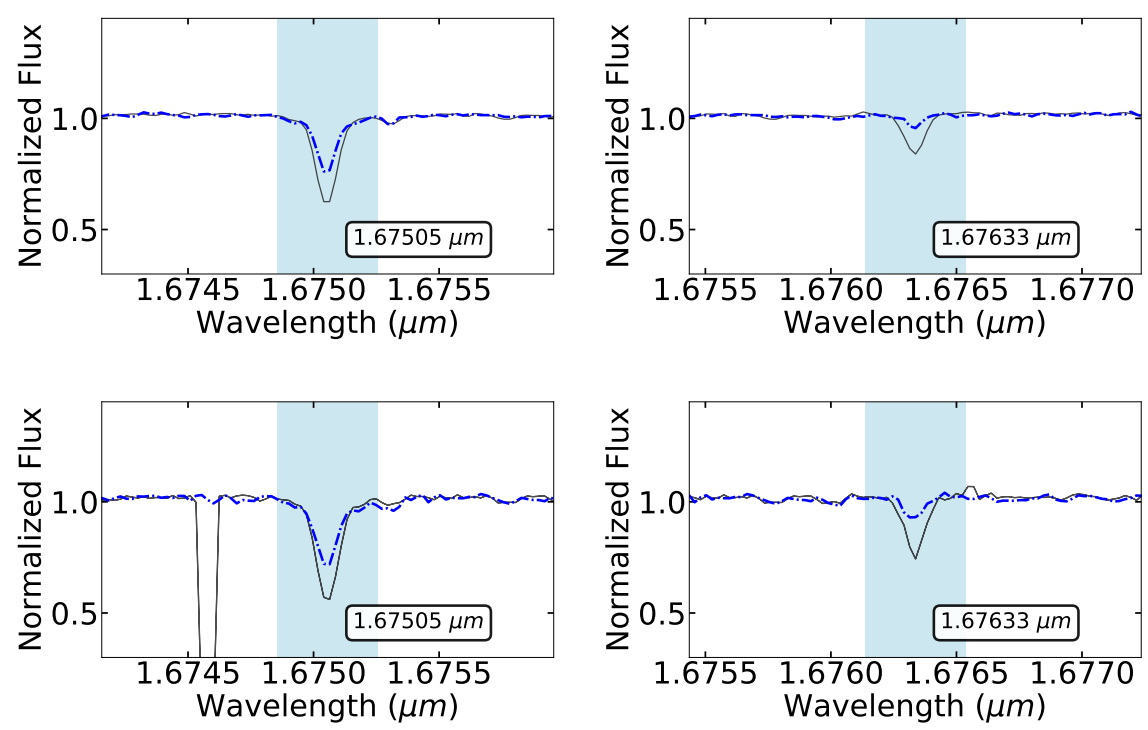

Fig. E.1. Comparison between the spectra of a normal (blue) and an Al-rich (black) star, with similar stellar parameters around Al I absorption lines at $1.67189,1.67505$ and $1.67633 \mu \mathrm{m}$. The last row shows the typical spectrum of a bulge NRS (blue) with low aluminium enrichment from Schiavon et al. (2017). 